\title{
Adult-onset Mild Encephalitis/Encephalopathy with a Reversible Splenial Lesion Induced by MRSA Endocarditis
}

\author{
Hideharu Hagiya, Fumio Otsuka \\ Department of General Medicine, Okayama University Graduate School of Medicine, Dentistry and Pharmaceutical Sciences, Okayama, Japan
}

Received: $11 / 01 / 2022$

Accepted: 01/02/2022

Published: 25/02/2022

How to cite this article: Hagiya H, Otsuka F. Adult-onset mild encephalitis/encephalopathy with a reversible splenial lesion induced by MRSA endocarditis. EJCRIM 2022;9:doi:10.12890/2022_003185.

Conflicts of Interests: The Authors declare that there are no competing interests.

This article is licensed under a Commons Attribution Non-Commercial 4.0 License

\section{ABSTRACT}

Background: Mild encephalitis/encephalopathy and a reversible splenial lesion (MERS) is a clinicoradiological syndrome with an unknown pathogenic mechanism, which usually involves children. Thus, adult-onset MERS is quite rare.

Case presentation: A 71-year-old man, undergoing haemodialysis due to diabetes-induced chronic kidney disease, manifested a persistent fever and disorientation. Blood culture detected methicillin-resistant Staphylococcus aureus (MRSA), while echocardiography revealed vegetation in the aortic and mitral valves. Magnetic resonance imaging of the head revealed a fluid-attenuated inversion recovery-high, diffusion-weighted image-high lesion in the splenium of the corpus callosum, with a number of emboli. Accordingly, the patient was diagnosed with MERS induced by MRSA endocarditis.

Discussion: Neurological impairment by MERS can be reversible. However, the differential diagnosis of the disease includes ischaemic lesions, multiple sclerosis, malignant lymphoma, acute disseminated encephalomyelitis, and posterior reversible encephalopathy. Clinicians should consider these diseases when MERS is suspected.

\section{KEYWORDS}

Bacteraemia, corpus callosum, infective endocarditis, mild encephalitis/encephalopathy with a reversible splenial lesion, Staphylococcus aureus

\section{LEARNING POINTS}

- Adult-onset mild encephalitis/encephalopathy and a reversible splenial lesion (MERS) is quite rare, and physicians should be aware of it as a differential diagnosis of a diffusion-weighted image-high lesion in the splenium of the corpus callosum.

- Methicillin-resistant Staphylococcus aureus (MRSA) has rarely been reported as a triggering factor for MERS.

\section{INTRODUCTION}

Mild encephalitis/encephalopathy with a reversible splenial lesion (MERS), first reported by Japanese paediatricians in 2004 [1, 2], is a clinicoradiological syndrome with an unknown pathogenic mechanism. MERS patients transiently develop delirium, impaired consciousness and seizures, which reportedly subside within a month without specific treatment. The disease typically affects children, and adult-onset MERS is an uncommon condition ${ }^{[3]}$. We herein describe a case of adult-onset MERS induced by methicillin-resistant Staphylococcus aureus (MRSA) endocarditis. 


\section{CASE DESCRIPTION}

A 71-year-old man, undergoing haemodialysis due to diabetes-induced chronic kidney disease, manifested a persistent fever. He was disoriented (Japan coma scale: II-10), and head magnetic resonance imaging (MRI) revealed multiple bilateral emboli. Blood culture detected MRSA, while echocardiography revealed vegetation in the aortic (16 mm) and mitral (13 mm) valves. The patient was diagnosed with infective endocarditis and was referred to our medical institute.

\section{Methods and procedures}

A follow-up head MRI revealed a newly emerging, fluid-attenuated inversion recovery-high, diffusion-weighted image-high lesion in the splenium of the corpus callosum, with increased emboli (Fig. 1). Based on these characteristic findings, the patient was diagnosed with MERS induced by MRSA endocarditis. The patient underwent emergent double valve replacement and intensive antimicrobial therapy. His consciousness gradually recovered postoperatively, and he was discharged without neurological sequelae.
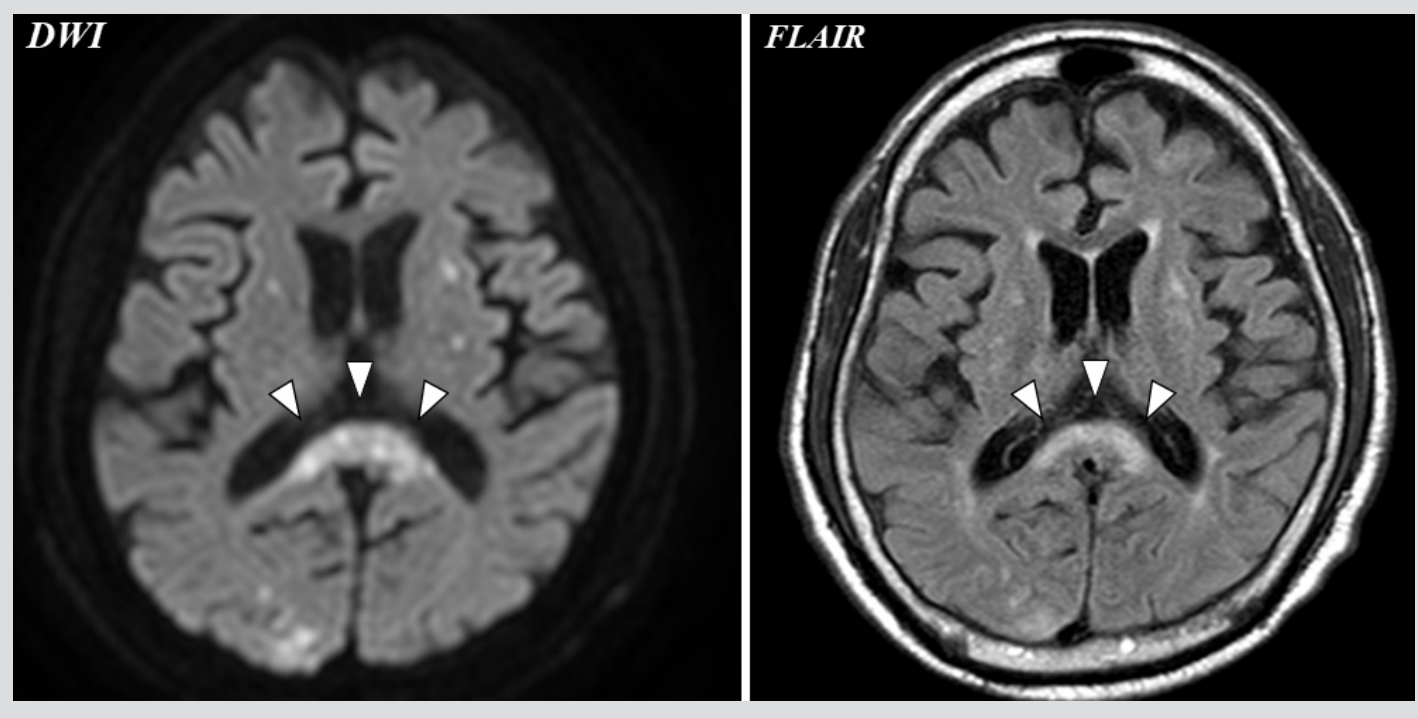

Figure 1. Head magnetic resonance images showing a typical finding of mild encephalitis/encephalopathy with a reversible splenial lesion (MERS, arrowheads). DWI, diffusion-weighted image; FLAIR, fluid-attenuated inversion recovery

\section{DISCUSSION}

The typical MRI findings of MERS include high intensity on T2-weighted image, fluid-attenuated inversion recovery image, and diffusionweighted image, and hyper-isointensity on T1-weighted image ${ }^{[1]}$. Based on its features, MERS is characterised as MERS type I, which mostly involves a single lesion in the midline of the splenium of the corpus callosum, and MERS type II, which demonstrates abnormal high intensity at symmetrical cerebral white matter lesions or the anterior part of the corpus callosum ${ }^{[4]}$. The present case was considered MERS type I according to the MRI findings.

Various infectious aetiologies reportedly trigger MERS. However, S. aureus infection-associated MERS has rarely been described in the literature ${ }^{[5]}$. Thus, this case of adult-onset MERS induced by MRSA endocarditis could be quite rare. The differential diagnosis of MERS includes ischaemic lesions, multiple sclerosis, malignant lymphoma, acute disseminated encephalomyelitis, and posterior reversible encephalopathy ${ }^{[6]}$. Clinicians should consider these diseases when MERS is suspected.

\section{REFERENCES}

1. Tada H, Takanashi J, Barkovich AJ, Oba H, Maeda M, Tsukahara H, et al. Clinically mild encephalitis/encephalopathy with a reversible splenial lesion. Neurology 2004;63:18541858.

2. Takanashi J. Two newly proposed infectious encephalitis/encephalopathy syndromes. Brain Dev 2009;31:521-528.

3. Yuan J, Yang S, Wang S, Qin W, Yang L, Hu W. Mild encephalitis/encephalopathy with reversible splenial lesion (MERS) in adults-a case report and literature review. BMC Neurol 2017;17:103.

4. Takanashi J, Barkovich A, Shiihara T, Tada H, Kawatani M, Tsukahara H, et al. Widening spectrum of a reversible splenial lesion with transiently reduced diffusion. Am J Neuroradiol 2006;27:836-838.

5. Anada R, Nukui T, Hayashi T, Konishi H, Dougu N, Nakatsuji Y. A case of clinically mild encephalitis/encephalopathy with a reversible splenial lesion (MERS) associated with infectious endocarditis caused by Staphylococcus aureus. Rinsho Shinkeigaku 2019;59:666-668 [in Japanese].

6. Pan JJ, Zhao YY, Lu C, Hu YH, Yang Y. Mild encephalitis/encephalopathy with a reversible splenial lesion: five cases and a literature review. Neurol Sci 2015;36:2043-2051. 